Research Article

\title{
Skew Circulant Type Matrices Involving the Sum of Fibonacci and Lucas Numbers
}

\author{
Zhaolin Jiang and Yunlan Wei \\ School of Science, Linyi University, Shuangling Road, Linyi, Shandong 276000, China \\ Correspondence should be addressed to Zhaolin Jiang; jzh1208@sina.com
}

Received 27 June 2014; Revised 8 August 2014; Accepted 11 August 2014

Academic Editor: Yongli Song

Copyright (C) 2015 Z. Jiang and Y. Wei. This is an open access article distributed under the Creative Commons Attribution License, which permits unrestricted use, distribution, and reproduction in any medium, provided the original work is properly cited.

Skew circulant and circulant matrices have been an ideal research area and hot issue for solving various differential equations. In this paper, the skew circulant type matrices with the sum of Fibonacci and Lucas numbers are discussed. The invertibility of the skew circulant type matrices is considered. The determinant and the inverse matrices are presented. Furthermore, the maximum column sum matrix norm, the spectral norm, the Euclidean (or Frobenius) norm, the maximum row sum matrix norm, and bounds for the spread of these matrices are given, respectively.

\section{Introduction}

As is well-known, skew circulant and circulant matrices play a crucial role for solving various differential equations. Authors in [1] presented the skew circulant matrices as preconditioners for linear multistep formulae (LMF-)based ordinary differential equations (ODEs) codes. Claeyssen et al. [2] discussed factor block circulant and periodic solutions of undamped matrix differential equations. Using circulant matrix, Karasözen and Şimşek [3] considered periodic boundary conditions such that no additional boundary terms will appear after semidiscretization. Meyer and Rjasanow [4] have presented an effective direct solution method for certain boundary element equations in 3D. Guo et al. concerned on generic Dn-Hopf bifurcation to a delayed Hopfield-CohenGrossberg model of neural networks (5.17) in [5], where $T$ denoted an interconnection matrix. In particular, they assumed that $T$ is a symmetric circulant matrix. In [6], Jin et al. proposed the GMRES method with the Strang-type block-circulant preconditioner for solving singular perturbation delay differential equations. In [7], two new normalform realizations are presented which utilize circulant and skew circulant matrices as their state transition matrices. The well known second-order coupled form is a special case of the skew circulant form. Compared with cyclic convolution algorithm, the skew cyclic convolution algorithm [8] is able to perform filtering procedure in approximately half of computational cost for real signals. In [9], a new fast algorithm for optimal design of block digital filters (BDFs) was proposed based on skew circulant matrix. Spectral decompositions of skew circulant and skew left circulant matrices were discussed in [10]. Li et al. [11] gave the style spectral decomposition of skew circulant matrix firstly and then dealt with the optimal backward perturbation analysis for the linear system with skew circulant coefficient matrix.

Some scholars have given various algorithms for the determinants and inverses of nonsingular circulant matrices $[12,13]$. Unfortunately, the computational complexity of these algorithms is very amazing with the order of matrix increasing. However, some authors gave the explicit determinants and inverse of circulant and skew circulant involving some famous numbers. For example, Shen et al. considered circulant matrices with Fibonacci and Lucas numbers and presented their explicit determinants and inverses by constructing the transformation matrices in [14]. Gao et al. [15] gave explicit determinants and inverses of skew circulant and skew left circulant matrices with Fibonacci and Lucas numbers. In [16], Jiang et al. discussed the nonsingularity of the skew circulant type matrices and presented explicit determinants and inverse matrices of these special matrices. Furthermore, four kinds of norms and bounds for the spread of these matrices are given separately. In [17], Jiang and 
Hong give exact determinants of some special circulant matrices involving four kinds of famous numbers. Authors [18] discussed the nonsingularity of the circulant type matrix and presented the explicit determinant and inverse matrices.

There are several papers on the norms of some special matrices. Solak [19] established the lower and upper bounds for the spectral norms of circulant matrices with classical Fibonacci and Lucas numbers entries. İpek [20] investigated an improved estimation for spectral norms of these matrices.

Beginning with Mirsky [21] several authors [22-24] have obtained bounds for the spread of a matrix.

Additionally, skew circulant type matrices include skew circulant and skew left circulant matrices. The norm and spread of skew circulant type matrices have not been studied. It is hoped that this paper will help in changing this. More work continuing the present paper is forthcoming.

The sum of Fibonacci and Lucas sequence is defined by the following recurrence relations:

$$
\mathbf{R}_{n+2}=\mathbf{Q}_{n+1}+\mathbf{Q}_{n} \quad \text { where } \boldsymbol{Q}_{0}=2, \mathbf{Q}_{1}=2,
$$

for $n \geq 0$. The first few values of the sequence are given by the following table:

$$
\begin{array}{c|ccccccccc}
n & 0 & 1 & 2 & 3 & 4 & 5 & 6 & 7 & 8 \\
\hline \mathfrak{\Omega}_{n} & 2 & 2 & 4 & 6 & 10 & 16 & 26 & 42 & 68
\end{array} .
$$

The $\left\{\boldsymbol{\Omega}_{n}\right\}$ is given by the formula

$$
\mathbf{\Omega}_{n}=\frac{\alpha^{n-1}-\beta^{n-1}}{\alpha-\beta}+\frac{\alpha^{n}-\beta^{n}}{\alpha-\beta}+\frac{\alpha^{n+1}-\beta^{n+1}}{\alpha-\beta},
$$

where $\alpha$ and $\beta$ are the roots of the characteristic equation $x^{2}-$ $x-1=0$.

In this paper, we consider skew circulant type matrices, including the skew circulant and skew left circulant matrices.

We define a sum of Fibonacci and Lucas skew circulant matrix which is an $n \times n$ complex matrix with following form:

$$
\begin{aligned}
& \operatorname{SCirc}\left(\mathfrak{\Omega}_{1}, \mathbf{\Omega}_{2}, \ldots, \boldsymbol{\Omega}_{n}\right) \\
& =\left(\begin{array}{ccccc}
\mathfrak{Q}_{1} & \mathfrak{Q}_{2} & \cdots & \mathfrak{Q}_{n-1} & \mathfrak{Q}_{n} \\
-\mathfrak{Q}_{n} & \mathfrak{Q}_{1} & \cdots & \mathfrak{Q}_{n-2} & \mathfrak{Q}_{n-1} \\
\vdots & \vdots & \ddots & \vdots & \vdots \\
-\mathfrak{Q}_{3} & -\mathfrak{Q}_{4} & \cdots & \mathfrak{Q}_{1} & \mathfrak{Q}_{2} \\
-\mathfrak{Q}_{2} & -\mathfrak{Q}_{3} & \cdots & -\mathfrak{Q}_{n} & \mathfrak{Q}_{1}
\end{array}\right),
\end{aligned}
$$

where each row is a cyclic shift of the row above the right.

Besides, a sum of Fibonacci and Lucas skew left circulant matrix is given by

$$
\begin{aligned}
& \operatorname{SLCirc}\left(\mathfrak{Q}_{1}, \mathfrak{\Omega}_{2}, \ldots, \mathfrak{\Omega}_{n}\right) \\
& =\left(\begin{array}{ccccc}
\mathfrak{Q}_{1} & \mathfrak{Q}_{2} & \cdots & \mathfrak{Q}_{n-1} & \mathfrak{Q}_{n} \\
\mathfrak{L}_{2} & \mathfrak{Q}_{3} & \cdots & \mathfrak{Q}_{n} & -\mathfrak{Q}_{1} \\
\vdots & \vdots & \ddots & \vdots & \vdots \\
\mathfrak{Q}_{n-1} & \mathfrak{Q}_{n} & \cdots & -\mathfrak{Q}_{n-3} & -\mathfrak{Q}_{n-2} \\
\mathfrak{Q}_{n} & -\mathfrak{Q}_{1} & \cdots & -\mathfrak{Q}_{n-2} & -\mathfrak{Q}_{n-1}
\end{array}\right) \text {, }
\end{aligned}
$$

where each row is a cyclic shift of the row above the left.
Lemma 1. Let $\left\{\boldsymbol{\Omega}_{n}\right\}$ be the sum of Fibonacci and lucas numbers; then,

$$
\begin{aligned}
\text { (i) } \sum_{i=1}^{n} \mathfrak{\Omega}_{i} & =\mathfrak{\Omega}_{n+2}-4, \\
\text { (ii) } \sum_{i=1}^{n} \mathfrak{\Omega}_{i}^{2} & =\mathfrak{\Omega}_{n} \mathfrak{\Omega}_{n+1}-4,
\end{aligned}
$$

$$
\text { (iii) } \sum_{i=1}^{n} i \mathbf{\Omega}_{i}=(n-2) \mathbf{\Omega}_{n+2}+\mathbf{\Omega}_{n}+6 \text {. }
$$

Proof. According to

$$
\mathfrak{Q}_{n+1}=\mathfrak{Q}_{n}+\mathfrak{Q}_{n-1} \quad \text { where } \mathfrak{Q}_{0}=2, \mathfrak{Q}_{1}=2,
$$

we have

$$
\begin{aligned}
\sum_{i=1}^{n} \mathfrak{Q}_{i}= & \mathfrak{Q}_{1}+\mathfrak{\Omega}_{2}+\cdots+\mathfrak{\Omega}_{n} \\
= & \left(\mathfrak{Q}_{3}-\mathfrak{\Omega}_{2}\right)+\left(\mathfrak{Q}_{4}-\mathfrak{\Omega}_{3}\right) \\
& +\cdots+\left(\mathfrak{Q}_{n+2}-\mathfrak{Q}_{n+1}\right) \\
= & \mathfrak{Q}_{n+2}-\mathfrak{\Omega}_{2},
\end{aligned}
$$

$$
\begin{aligned}
& \sum_{i=1}^{n} \mathfrak{R}_{i}^{2}=\mathfrak{Q}_{1}^{2}+\mathfrak{Q}_{2}^{2}+\cdots+\mathfrak{Q}_{n}^{2} \\
& =\mathfrak{Q}_{1}\left(\mathfrak{Q}_{2}-\mathfrak{Q}_{0}\right)+\mathfrak{Q}_{2}\left(\mathfrak{Q}_{3}-\mathfrak{Q}_{1}\right) \\
& +\cdots+\mathfrak{\Omega}_{n}\left(\mathfrak{Q}_{n+1}-\mathfrak{Q}_{n-1}\right) \\
& =\mathfrak{Q}_{n} \mathfrak{Q}_{n+1}-\mathfrak{Q}_{0} \mathfrak{Q}_{1},
\end{aligned}
$$$$
\sum_{i=1}^{n} i \mathfrak{Q}_{i}=\mathfrak{\Omega}_{1}+2 \mathfrak{\Omega}_{2}+\cdots+n \mathfrak{Q}_{n}
$$

$$
\begin{aligned}
= & \left(\mathfrak{Q}_{2}-\mathfrak{Q}_{0}\right)+2\left(\mathfrak{Q}_{2}-\mathfrak{Q}_{0}\right) \\
& +\cdots+n\left(\mathfrak{Q}_{n+1}-\mathfrak{Q}_{n-1}\right) \\
= & -2 \sum_{i=1}^{n} \mathfrak{Q}_{i}+(n+1) \mathfrak{Q}_{n}+n \mathbf{Q}_{n+1}-\mathbf{Q}_{0} \\
= & (n-2) \mathfrak{Q}_{n+2}+\mathfrak{Q}_{n}+2 \mathfrak{Q}_{2}-\mathfrak{Q}_{0},
\end{aligned}
$$

and hence

$$
\begin{aligned}
& \sum_{i=1}^{n} \mathfrak{Q}_{i}=\mathfrak{Q}_{n+2}-4, \\
& \sum_{i=1}^{n} \mathfrak{\Omega}_{i}^{2}=\mathfrak{Q}_{n} \mathfrak{\Omega}_{n+1}-4, \\
& \sum_{i=1}^{n} i \mathfrak{Q}_{i}=(n-2) \mathfrak{\Omega}_{n+2}+\mathfrak{\Omega}_{n}+6 .
\end{aligned}
$$

This completes the proof. 
Lemma 2 (see $[10])$. Let $A=\operatorname{SLCirc}\left(a_{1}, a_{2}, \ldots, a_{n}\right)$ be a skew left circulant matrix and let $n$ be odd; then,

$$
\begin{gathered}
\lambda_{j}= \pm\left|\sum_{k=1}^{n} a_{k} \omega^{(j-(1 / 2))(k-1)}\right| \quad\left(j=1,2, \ldots, \frac{n-1}{2}\right) \\
\lambda_{(n+1) / 2}=\sum_{k=1}^{n}\left|a_{k}(-1)^{k-1}\right|,
\end{gathered}
$$

where $\lambda_{j}(j=1,2, \ldots,(n-1) / 2,(n+1) / 2)$ are the eigenvalues of $A$.

\section{Determinant and Inverse of Skew Circulant Matrix with the Sum of Fibonacci and Lucas Numbers}

In this section, let $A_{n}=\operatorname{SCirc}\left(\mathfrak{\Omega}_{1}, \ldots, \mathfrak{\Omega}_{n}\right)$ be a skew circulant matrix. First of all, a determinant explicit formula for the matrix $A_{n}$ is given. After that, we prove that $A_{n}$ is an invertible matrix for any positive interger $n$, and then we find the inverse of the matrix $A_{n}$. In the following, let

$$
\begin{aligned}
& x=-\frac{2+\mathfrak{Q}_{n}}{2+\mathfrak{Q}_{n+1}}, \quad t=2, \quad c=2+\mathfrak{Q}_{n+1}, \\
& d=2+\mathfrak{Q}_{n}, \quad l_{n}^{\prime}=\sum_{k=1}^{n-1} \mathfrak{Q}_{k+1} \cdot x^{n-(k+1)} \quad(n \geq 2), \\
& l_{n}=2+t \mathfrak{\Omega}_{n}+\sum_{k=1}^{n-2}\left(t \mathbf{\Omega}_{k+1}-\mathfrak{\Omega}_{k+2}\right) \cdot x^{n-(k+1)} \quad(n \geq 3) .
\end{aligned}
$$

Theorem 3. Let $A_{n}=\operatorname{SCirc}\left(\mathfrak{Q}_{1}, \ldots, \mathfrak{Q}_{n}\right)$ be a skew circulant matrix; then

$$
\operatorname{det} A_{n}=2\left[2+t \mathbf{\Omega}_{n}+\sum_{i=1}^{n-2}\left(t \mathbf{\Omega}_{i+1}-\mathbf{\Omega}_{i+2}\right) x^{n-(i+1)}\right] \cdot c^{n-2}
$$$$
(n \geq 3) \text {, }
$$

where $\mathbb{2}_{n}$ is the nth sum of Fibonacci andLucas numbers.
Proof. Obviously, $\operatorname{det} A_{3}=304$ satisfies the equation. In the case $n>3$, let

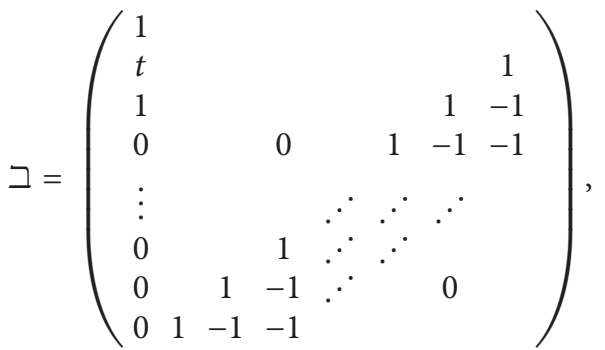

$$
\begin{aligned}
& \Omega_{1}=\left(\begin{array}{cccccc}
1 & 0 & 0 & \cdots & 0 & 0 \\
0 & x^{n-2} & 0 & \cdots & 0 & 1 \\
0 & x^{n-3} & 0 & \cdots & 1 & 0 \\
\vdots & \vdots & \vdots & \ddots & \vdots & \vdots \\
0 & x & 1 & \cdots & 0 & 0 \\
0 & 1 & 0 & \cdots & 0 & 0
\end{array}\right)
\end{aligned}
$$

be two $n \times n$ matrices; then, we have

$$
\beth A_{n} \Omega_{1}=\left(\begin{array}{cccccc}
2 & l_{n}^{\prime} & c_{13} & \cdots & c_{1, n-1} & c_{1 n} \\
0 & l_{n} & c_{23} & \cdots & c_{2, n-1} & c_{2 n} \\
0 & 0 & c & & & \\
0 & 0 & d & \ddots & & \\
\vdots & \vdots & & \ddots & c & \\
0 & 0 & & & d & c
\end{array}\right),
$$

where

$$
\begin{aligned}
& c_{1 j}=\mathfrak{\Omega}_{n+2-j} \quad(j=3,4, \ldots, n), \\
& c_{2 j}=t \mathbf{\Omega}_{n+2-j}-\mathbf{\Omega}_{n+3-j} \quad(j=3,4, \ldots, n) .
\end{aligned}
$$

So it holds that

$$
\begin{aligned}
\operatorname{det} \beth \operatorname{det} A_{n} \operatorname{det} \Omega_{1}=2 & {\left[2+t \mathbf{\Omega}_{n}+\sum_{k=1}^{n-2}\left(t \mathbf{\Omega}_{k+1}-\mathbf{\Omega}_{k+2}\right)\right.} \\
& \left.\times x^{n-(k+1)}\right] \cdot\left(2+\mathbf{\Omega}_{n+1}\right)^{n-2} .
\end{aligned}
$$

While taking $\operatorname{det} \beth=\operatorname{det} \Omega_{1}=(-1)^{(n-1)(n-2) / 2}$, we have

$$
\begin{aligned}
\operatorname{det} A_{n}=2 & {\left[2+t \mathfrak{\Omega}_{n}+\sum_{k=1}^{n-2}\left(t \mathfrak{\Omega}_{k+1}-\mathfrak{\Omega}_{k+2}\right)\right.} \\
& \left.\times x^{n-(k+1)}\right] \cdot\left(2+\mathfrak{\Omega}_{n+1}\right)^{n-2} .
\end{aligned}
$$

This completes the proof.

Theorem 4. Let $A_{n}=\operatorname{SCirc}\left(\mathfrak{\Omega}_{1}, \ldots, \mathfrak{\Omega}_{n}\right)$ be a skew circulant matrix; then $A_{n}$ is an invertible matrix for any positive interger $n$.

Proof. Taking $n=1$ in Theorem 3, we have $\operatorname{det} A_{1} \neq$ 0 . Hence $A_{1}$ is invertible. In the case $n>1$, since 
$\mathfrak{Q}_{n}=\left(\alpha^{n-1}-\beta^{n-1}\right) /(\alpha-\beta)+\left(\alpha^{n}-\beta^{n}\right) /(\alpha-\beta)+\left(\alpha^{n+1}-\right.$ $\left.\beta^{n+1}\right) /(\alpha-\beta)$, where $\alpha+\beta=1, \alpha \beta=-1$, we have

$$
\begin{aligned}
f\left(\omega^{k} \eta\right)= & \sum_{j=1}^{n} \mathfrak{Q}_{j}\left(\omega^{k} \eta\right)^{j-1} \\
= & \sum_{j=1}^{n}\left(\frac{\alpha^{j-1}-\beta^{j-1}}{\alpha-\beta}+\frac{\alpha^{j}-\beta^{j}}{\alpha-\beta}\right. \\
& \left.+\frac{\alpha^{j+1}-\beta^{j+1}}{\alpha-\beta}\right)\left(\omega^{k} \eta\right)^{j-1} \\
= & \sum_{j=1}^{n}\left(\frac{\alpha^{j-1}+\alpha^{j}+\alpha^{j+1}}{\alpha-\beta}\right)\left(\omega^{k} \eta\right)^{j-1} \\
& -\sum_{j=1}^{n}\left(\frac{\beta^{j-1}+\beta^{j}+\beta^{j+1}}{\alpha-\beta}\right)\left(\omega^{k} \eta\right)^{j-1} \\
= & \frac{1+\alpha+\alpha^{2}}{\alpha-\beta} \frac{1+\alpha^{n}}{1-\alpha \omega^{k} \eta} \\
= & \frac{1+\beta+\beta^{2}}{\alpha-\beta} \frac{1+\beta^{n}}{1-\beta \omega^{k} \eta} \\
& 2+\mathfrak{Q}_{n+1}+\left(2+\mathfrak{Q}_{n}\right) \omega^{k} \eta \\
1-\omega^{k} \eta-\omega^{2 k} \eta^{2} & (k=1,2, \ldots, n-1),
\end{aligned}
$$

where $\omega=\exp (2 \pi i / n), \eta=\exp (\pi i / n)$. If there exists $\omega^{l} \eta(l=$ $1,2, \ldots, n-1)$ such that $f\left(\omega^{l} \eta\right)=0$, we have $2+\mathfrak{Q}_{n+1}+(2+$ $\left.\mathfrak{\Omega}_{n}\right) \omega^{l} \eta=0$ for $1-\omega^{l} \eta-\omega^{2 l} \eta^{2} \neq 0$, and hence it follows that $\omega^{l} \eta=-\left(2+\mathfrak{Q}_{n+1}\right) /\left(2+\mathfrak{Q}_{n}\right)$ is a real number. Since

$$
\begin{aligned}
\omega^{l} \eta & =\exp \left(\frac{(2 l+1) \pi i}{n}\right) \\
& =\cos \frac{(2 l+1) \pi}{n}+i \sin \frac{(2 l+1) \pi}{n},
\end{aligned}
$$

it yields that $\sin ((2 l+1) \pi) / n=0$, so we have $\omega^{l} \eta=-1$ for $0<$ $(2 l+1) \pi / n<2 \pi$. Since $x=-1$ is not the root of the equation $2+\mathfrak{Q}_{n+1}+\left(2+\mathfrak{Q}_{n}\right) x=0(n \geq 1)$. We obtain $f\left(\omega^{k} \eta\right) \neq 0$ for any $\omega^{k} \eta(k=1,2, \ldots, n-1)$, while

$$
\begin{aligned}
f(\eta) & =\sum_{j=1}^{n} \mathfrak{Q}_{j} \eta^{j-1} \\
& =\frac{2+\mathfrak{\Omega}_{n+1}+\left(2+\mathfrak{\Omega}_{n}\right) \eta}{1-\eta-\eta^{2}} \neq 0 .
\end{aligned}
$$

It follows from Lemma 1 in [25] that the conclusion holds.

Lemma 5. Let the matrix $\mathscr{H}=\left[h_{i j}\right]_{i, j=1}^{n-2}$ be of the form

$$
h_{i j}= \begin{cases}2+\mathfrak{Q}_{n+1}, & i=j, \\ 2+\mathfrak{Q}_{n}, & i=j+1, \\ 0, & \text { otherwise. }\end{cases}
$$

Then the inverse $\mathscr{H}^{-1}=\left[h_{i, j}^{\prime}\right]_{i, j=1}^{n-2}$ of the matrix $\mathscr{H}$ is equal to

$$
h_{i j}^{\prime}= \begin{cases}\frac{(-d)^{i-j}}{c^{i-j+1}}, & i \geq j, \\ 0, & i<j .\end{cases}
$$

Proof. Let $e_{i j}=\sum_{k=1}^{n-2} h_{i k} h_{k j}^{\prime}$. Obviously, $e_{i j}=0$ for $i<j$. In the case $i=j$, we obtain $e_{i i}=h_{i i} h_{i i}^{\prime}=\left(2+\mathfrak{Q}_{n+1}\right) \cdot 1 /\left(2+\mathfrak{Q}_{n+1}\right)=1$. For $i \geq j+1$, we obtain

$$
\begin{aligned}
e_{i j} & =\sum_{k=1}^{n-2} h_{i k} h_{k j}^{\prime}=h_{i, i-1} h_{i-1, j}^{\prime}+h_{i i} h_{i j}^{\prime} \\
& =d \cdot \frac{(-d)^{i-j-1}}{c^{i-j}}+c \cdot \frac{(-d)^{i-j}}{c^{i-j+1}}=0 .
\end{aligned}
$$

Hence, we get $\mathscr{H} \mathscr{H}^{-1}=I_{n-2}$; here $I_{n-2}$ is $(n-2) \times(n-2)$ identity matrix. Similarly, we can verify $\mathscr{H}^{-1} \mathscr{H}=I_{n-2}$. Thus, the proof is completed.

Theorem 6. Let $A_{n}=\operatorname{SCirc}\left(\mathfrak{Q}_{1}, \ldots, \mathfrak{\Omega}_{n}\right)$ be a skew circulant matrix; then

$$
\left(A_{n}\right)^{-1}=\frac{1}{l_{n}} \cdot \operatorname{SCirc}\left(y_{1}^{\prime}, y_{2}^{\prime}, \ldots, y_{n}^{\prime}\right) \quad(n \geq 4),
$$

where

$$
\begin{aligned}
& y_{1}^{\prime}=1-\left[(6-4 t) \cdot \frac{(-d)^{n-3}}{c^{n-2}}\right. \\
& \left.+\sum_{i=1}^{n-3}\left(\mathbf{\Omega}_{n+2-i}-t \mathbf{\Omega}_{n+1-i}\right) \cdot \frac{(-d)^{i-1}}{c^{i}}\right] \\
& (n \geq 4) \text {, } \\
& y_{2}^{\prime}=-t-\sum_{i=1}^{n-2}\left(\boldsymbol{\Omega}_{n+1-i}-t \boldsymbol{\Omega}_{n-i}\right) \cdot \frac{(-d)^{i-1}}{c^{i}}, \\
& y_{3}^{\prime}=-(6-4 t) \cdot \frac{1}{c}, \\
& y_{4}^{\prime}=-\sum_{i=1}^{2}\left(\mathbf{\Omega}_{1+i}-t \mathbf{\Omega}_{i}\right) \cdot \frac{(-d)^{i-1}}{c^{i}}, \\
& y_{k}^{\prime}=-\sum_{i=1}^{2}\left(\boldsymbol{\Omega}_{1+i}-t \mathbf{\Omega}_{r+i}\right) \cdot \frac{(-d)^{k-5+i}}{c^{k-4+i}} \\
& (k=5,6, \ldots, n) .
\end{aligned}
$$

Proof. Let

$$
\Omega_{2}=\left(\begin{array}{cccccc}
1 & -\frac{l_{n}^{\prime}}{2} & \omega_{13} & \omega_{14} & \cdots & \omega_{1 n} \\
0 & 1 & \omega_{23} & \omega_{24} & \cdots & \omega_{2 n} \\
0 & 0 & 1 & 0 & \cdots & 0 \\
0 & 0 & 0 & 1 & \cdots & 0 \\
\vdots & \vdots & \vdots & \vdots & \ddots & \vdots \\
0 & 0 & 0 & 0 & \cdots & 1
\end{array}\right),
$$


where

$$
\begin{gathered}
\omega_{1 j}=\frac{1}{2}\left[\frac{l_{n}^{\prime}}{l_{n}}\left(t \mathfrak{\Omega}_{n+2-j}-\mathfrak{Q}_{n+3-j}\right)-\mathfrak{Q}_{n+2-j}\right] \\
(j=3,4, \ldots, n), \\
\omega_{2 j}=\frac{1}{l_{n}} \cdot\left(\mathfrak{\Omega}_{n+3-j}-t \mathfrak{\Omega}_{n+2-j}\right) \quad(j=3,4, \ldots, n) .
\end{gathered}
$$

Then we have

$$
\neg A_{n} \Omega_{1} \Omega_{2}=\left(\begin{array}{cccccc}
2 & 0 & 0 & 0 & \cdots & 0 \\
0 & l_{n} & 0 & 0 & \cdots & 0 \\
0 & 0 & c & 0 & \cdots & 0 \\
0 & 0 & d & c & \cdots & 0 \\
\vdots & \vdots & \vdots & \vdots & \ddots & \vdots \\
0 & 0 & 0 & 0 & \cdots & c
\end{array}\right),
$$

so $\beth A_{n} \Omega_{1} \Omega_{2}=\mathscr{D} \oplus \mathscr{H}$, and here $D=\operatorname{diag}\left(2, l_{n}\right)$ is a diagonal matrix, and $\mathscr{D} \oplus \mathscr{H}$ is the direct sum of $\mathscr{D}$ and $\mathscr{H}$. If we denote $\Omega=\Omega_{1} \Omega_{2}$, then we obtain $A_{n}^{-1}=\Omega\left(\mathscr{D}^{-1} \oplus\right.$ $\left.\mathscr{H}^{-1}\right) \Sigma$. Since the last row elements of the matrix $\Omega$ are $\left(0,1, \omega_{23}, \omega_{24}, \ldots, \omega_{2, n-1}, \omega_{2 n}\right)$, the last row elements of the matrix $\Omega\left(\mathscr{D}^{-1} \oplus \mathscr{H}^{-1}\right)$ are $\left(0,1 / l_{n}, T_{23}, T_{24}, \ldots, T_{2 n}\right)$, where

$$
\begin{aligned}
T_{23} & =\sum_{i=1}^{n-2} \omega_{2,2+i} \cdot \frac{(-d)^{i-1}}{c^{i}} \quad(n \geq 3), \\
T_{2 k} & =\sum_{i=1}^{n+1-k} \omega_{2, k-1+i} \cdot \frac{(-d)^{i-1}}{c^{i}} \quad(k=3,4, \ldots, n) .
\end{aligned}
$$

Hence it follows from Lemma 5 that by letting $A_{n}^{-1}=$ $\operatorname{SCirc}\left(y_{1}, y_{2}, \ldots, y_{n}\right)$ then its last row elements are $\left(-y_{2}\right.$, $\left.-y_{3}, \ldots,-y_{n}, y_{1}\right)$ which are given by the following equations:

$$
\begin{aligned}
-y_{2} & =\frac{t}{l_{n}}+T_{23} \\
& =\frac{t}{l_{n}}+\frac{1}{l_{n}} \sum_{i=1}^{n-2}\left(\mathfrak{\Omega}_{n+1-i}-t \boldsymbol{\Omega}_{n-i}\right) \cdot \frac{(-d)^{i-1}}{c^{i}}, \\
-y_{3} & =T_{2, n}=\frac{1}{l_{n}}(6-4 t) \cdot \frac{1}{c} \\
-y_{4} & =T_{2, n-1}-T_{2 n} \\
& =\frac{1}{l_{n}} \sum_{i=1}^{2}\left(\mathfrak{\Omega}_{1+i}-t \mathfrak{\Omega}_{i}\right) \cdot \frac{(-d)^{i-1}}{c^{i}}, \\
-y_{5} & =T_{2, n-2}-T_{2 n-1}-T_{2 n} \\
& =\frac{1}{l_{n}} \sum_{i=1}^{2}\left(\mathfrak{\Omega}_{1+i}-t \mathfrak{\Omega}_{i}\right) \cdot \frac{(-d)^{i}}{c^{i+1}},
\end{aligned}
$$

$$
\begin{aligned}
-y_{k}= & T_{2, n-k+3}-T_{2, n-k+4}-T_{2, n-k+5} \\
= & \frac{1}{l_{n}} \sum_{i=1}^{2}\left(\mathfrak{\Omega}_{1+i}-t \boldsymbol{\Omega}_{i}\right) \cdot \frac{(-d)^{k-5+i}}{c^{k-4+i}}, \\
& \vdots \\
-y_{n}= & T_{23}-T_{24}-T_{25} \\
= & \sum_{i=1}^{n-2} \omega_{2,2+i} \cdot \frac{(-d)^{i-1}}{c^{i}}-\sum_{i=1}^{n-3} \omega_{2,3+i} \cdot \frac{(-d)^{i-1}}{c^{i}} \\
& -\sum_{i=1}^{n-4} \omega_{2,4+i} \cdot \frac{(-d)^{i-1}}{c^{i}} \\
= & \frac{1}{l_{n}} \sum_{i=1}^{2}\left(\boldsymbol{\Omega}_{1+i}-t \boldsymbol{\Omega}_{i}\right) \cdot \frac{(-d)^{n-5+i}}{c^{n-4+i}},
\end{aligned}
$$

$$
\begin{aligned}
y_{1}=\frac{1}{l_{n}}- & T_{23}- \\
=\frac{1}{l_{n}}-\frac{1}{l_{n}}[ & (6-4 t) \cdot \frac{(-d)^{n-3}}{c^{n-2}} \\
& \left.\quad+\sum_{i=1}^{n-3}\left(\mathbb{\Omega}_{n+2-i}-t \mathbb{\Omega}_{n+1-i}\right) \cdot \frac{(-d)^{i-1}}{c^{i}}\right] \\
& (n \geq 4) .
\end{aligned}
$$

Hence, we obtain

$$
\begin{aligned}
& y_{1}=\frac{1}{l_{n}}-\frac{1}{l_{n}}\left[(6-4 t) \cdot \frac{(-d)^{n-3}}{c^{n-2}}\right. \\
& \left.+\sum_{i=1}^{n-3}\left(\boldsymbol{\Omega}_{n+2-i}-t \boldsymbol{\Omega}_{n+1-i}\right) \frac{(-d)^{i-1}}{c^{i}}\right], \\
& y_{2}=-\frac{t}{l_{n}}-\frac{1}{l_{n}} \sum_{i=1}^{n-2}\left(\boldsymbol{\Omega}_{n+1-i}-t \boldsymbol{\Omega}_{n-i}\right) \cdot \frac{(-d)^{i-1}}{c^{i}}, \\
& y_{3}=-\frac{1}{l_{n}}(6-4 t) \cdot \frac{1}{c} \text {, } \\
& y_{4}=-\frac{1}{l_{n}} \sum_{i=1}^{2}\left(\boldsymbol{\Omega}_{1+i}-t \boldsymbol{\Omega}_{i}\right) \cdot \frac{(-d)^{i-1}}{c^{i}}, \\
& y_{5}=-\frac{1}{l_{n}} \sum_{i=1}^{2}\left(\mathfrak{\Omega}_{1+i}-t \boldsymbol{\Omega}_{i}\right) \cdot \frac{(-d)^{i}}{c^{i+1}},
\end{aligned}
$$




$$
\begin{gathered}
y_{k}=-\frac{1}{l_{n}} \sum_{i=1}^{2}\left(\mathfrak{\Omega}_{1+i}-t \mathfrak{\Omega}_{i}\right) \cdot \frac{(-d)^{k-5+i}}{c^{k-4+i}}, \\
\vdots \\
y_{n}=\frac{1}{l_{n}} \sum_{i=1}^{2}\left(\mathbf{\Omega}_{1+i}-t \mathbf{\Omega}_{i}\right) \cdot \frac{(-d)^{n-5+i}}{c^{n-4+i}}, \\
A_{n}^{-1}=\frac{1}{l_{n}} \cdot \operatorname{SCirc}\left(y_{1}^{\prime}, y_{2}^{\prime}, \ldots, y_{n}^{\prime}\right),
\end{gathered}
$$

$$
\begin{aligned}
\left(\left\|A_{n}\right\|_{F}\right)^{2} & =\sum_{i=1}^{n} \sum_{j=1}^{n}\left|a_{i j}\right|^{2} \\
& =n \sum_{i=1}^{n} \mathfrak{Q}_{i}^{2} \\
& =n\left(\mathfrak{Q}_{n} \mathfrak{Q}_{n+1}-4\right) .
\end{aligned}
$$

Thus

where

$$
\begin{aligned}
& y_{1}^{\prime}=1-\left[(6-4 t) \cdot \frac{(-d)^{n-3}}{c^{n-2}}\right. \\
& \left.+\sum_{i=1}^{n-3}\left(\mathbf{\Omega}_{n+2-i}-t \mathbf{\Omega}_{n+1-i}\right) \cdot \frac{(-d)^{i-1}}{c^{i}}\right] \\
& (n \geq 4) \text {, } \\
& y_{2}^{\prime}=-t-\sum_{i=1}^{n-2}\left(\mathbf{\Omega}_{n+1-i}-t \mathbf{\Omega}_{n-i}\right) \cdot \frac{(-d)^{i-1}}{c^{i}}, \\
& y_{3}^{\prime}=-(6-4 t) \cdot \frac{1}{c}, \\
& y_{4}^{\prime}=-\sum_{i=1}^{2}\left(\mathfrak{\Omega}_{1+i}-t \mathbf{\Omega}_{i}\right) \cdot \frac{(-d)^{i-1}}{c^{i}}, \\
& y_{k}^{\prime}=-\sum_{i=1}^{2}\left(\mathfrak{\Omega}_{1+i}-t \mathbf{\Omega}_{i}\right) \cdot \frac{(-d)^{k-5+i}}{c^{k-4+i}}, \quad(k=5,6, \ldots, n) .
\end{aligned}
$$

This completes the proof.

\section{Norm and Spread of Skew Circulant}

\section{Matrix with the Sum of Fibonacci and}

\section{Lucas Numbers}

Theorem 7. Let $A_{n}=\operatorname{SCirc}\left(\mathfrak{\Omega}_{1}, \ldots, \mathfrak{\Omega}_{n}\right)$ be a skew circulant matrix. The three kinds norms of $A_{n}$ are given by

$$
\begin{gathered}
\left\|A_{n}\right\|_{1}=\left\|A_{n}\right\|_{\infty}=\mathfrak{Q}_{n+2}-4, \\
\left\|A_{n}\right\|_{F}=\sqrt{n\left(\mathfrak{\Omega}_{n} \mathfrak{Q}_{n+1}-4\right) .}
\end{gathered}
$$

Proof. By Definition 4 in [26], (6), and (7), we have

$$
\begin{aligned}
\left\|A_{n}\right\|_{1} & =\left\|A_{n}\right\|_{\infty} \\
& =\sum_{i=1}^{n} \mathfrak{\Omega}_{i}=\mathbf{\Omega}_{n+2}-4,
\end{aligned}
$$

$$
\left\|A_{n}\right\|_{F}=\sqrt{n\left(\mathfrak{Q}_{n} \mathfrak{\Omega}_{n+1}-4\right)} .
$$

Theorem 8. Let $A_{n}^{\prime}=\operatorname{SCirc}\left(\mathfrak{Q}_{1},-\mathfrak{\Omega}_{2}, \ldots,-\mathfrak{\Omega}_{n-1}, \mathfrak{\Omega}_{n}\right)$ be an odd-order alternative skew circulant matrix and let $n$ be odd. Then

$$
\left\|A_{n}^{\prime}\right\|_{2}=\sum_{i=1}^{n} \mathfrak{Q}_{i}=\mathfrak{Q}_{n+2}-4 .
$$

Proof. By Lemma 1 in [25], we have

$$
\lambda_{j}\left(A_{n}^{\prime}\right)=\sum_{i=1}^{n}(-1)^{i-1} \boldsymbol{\Omega}_{i}\left(\omega^{j} \eta\right)^{i-1} .
$$

Therefore

$$
\begin{aligned}
\left|\lambda_{j}\left(A_{n}^{\prime}\right)\right| & \leq \sum_{i=1}^{n}\left|(-1)^{i-1} \mathfrak{Q}_{i}\right| \cdot\left|\left(\omega^{j} \eta\right)^{i-1}\right| \\
& =\sum_{i=1}^{n} \mathfrak{Q}_{i} \quad(j=0,1, \ldots, n-1) .
\end{aligned}
$$

Since $n$ is odd, $\sum_{i=1}^{n} \mathbf{Q}_{i}$ is an eigenvalue of $A_{n}^{\prime}$, which is

$$
\begin{gathered}
\left(\begin{array}{cccc}
\mathfrak{Q}_{1} & -\mathfrak{Q}_{2} & \cdots & \mathfrak{Q}_{n} \\
-\mathfrak{Q}_{n} & \mathfrak{Q}_{1} & \cdots & -\mathfrak{Q}_{n-1} \\
\mathfrak{Q}_{n-1} & -\mathfrak{Q}_{n} & \cdots & \mathfrak{Q}_{n-2} \\
\vdots & \vdots & \vdots & \vdots \\
\mathfrak{Q}_{2} & -\mathfrak{Q}_{3} & \cdots & \mathfrak{Q}_{1}
\end{array}\right)\left(\begin{array}{c}
1 \\
-1 \\
1 \\
-1 \\
\vdots \\
1
\end{array}\right) \\
=\sum_{i=1}^{n} \mathfrak{Q}_{i} \cdot\left(\begin{array}{c}
1 \\
-1 \\
1 \\
-1 \\
\vdots \\
1
\end{array}\right) .
\end{gathered}
$$

To sum up, we have

$$
\max _{0 \leq j \leq n-1}\left|\lambda_{j}\left(A_{n}^{\prime}\right)\right|=\sum_{i=1}^{n} \mathfrak{\Omega}_{i} .
$$

Since all skew circulant matrices are normal, by Lemma 7 in [26], (6), and (43), we have

$$
\left\|A_{n}^{\prime}\right\|_{2}=\sum_{i=1}^{n} \mathfrak{\Omega}_{i}=\mathfrak{\Omega}_{n+2}-4,
$$

which completes the proof. 
Theorem 9. Let $A_{n}=\operatorname{SCirc}\left(\mathfrak{Q}_{1}, \ldots, \mathfrak{Q}_{n}\right)$ be a skew circulant matrix; then the bounds for the spread of $A_{n}$ are

$$
\begin{gathered}
s\left(A_{n}\right) \leqslant \sqrt{2 n\left(\mathfrak{Q}_{n} \mathfrak{\Omega}_{n+1}-8\right)}, \\
s\left(A_{n}\right) \geq \frac{1}{n-1}\left|2 \mathfrak{Q}_{n+4}-n \mathfrak{\Omega}_{n+2}-6 n-20\right| .
\end{gathered}
$$

Proof. The trace of $A_{n}$ is denoted by $\operatorname{tr} A_{n}=n \Omega_{1}$. By Definition 5 in [26] and (36), we have

$$
s\left(A_{n}\right) \leqslant \sqrt{2 n\left(\mathfrak{Q}_{n} \mathfrak{Q}_{n+1}-8\right)} .
$$

Since

$$
\begin{aligned}
\sum_{i \neq j} a_{i j} & =\sum_{k=2}^{n}[n-(k-1)] \mathfrak{\Omega}_{k}-\sum_{k=2}^{n}(k-1) \mathfrak{\Omega}_{k} \\
& =(n+2) \sum_{k=2}^{n} \mathfrak{\Omega}_{k}-2 \sum_{k=2}^{n} k \mathfrak{\Omega}_{k},
\end{aligned}
$$

by (6) and (8),

$$
\begin{aligned}
\sum_{i \neq j} a_{i j} & =(n+2)\left(\mathfrak{Q}_{n+2}-6\right)-2\left(n \mathbf{Q}_{n+2}-\mathfrak{\Omega}_{n+3}-6+\mathbf{Q}_{4}\right) \\
& =2 \mathfrak{\Omega}_{n+4}-n \mathbf{\Omega}_{n+2}-6 n-20 .
\end{aligned}
$$

By Lemma 6 in [26], we have

$$
S\left(A_{n}\right) \geq \frac{1}{n-1}\left|2 \mathfrak{\Omega}_{n+4}-n \mathfrak{\Omega}_{n+2}-6 n-20\right| .
$$

\section{Determinant and Inverse of Skew Left Circulant Matrix with the Sum of Fibonacci and Lucas Numbers}

In this section, let $A_{n}^{\prime \prime}=\operatorname{SLCirc}\left(\mathfrak{\Omega}_{1}, \ldots, \mathfrak{\Omega}_{n}\right)$ be a skew left circulant matrix. By using the obtained conclusions in Section 2, we give a determinant explicit formula for the matrix $A_{n}^{\prime \prime}$. And then, we prove that $A_{n}^{\prime \prime}$ is an invertible matrix for any positive interger $n$. The inverse of the matrix $A_{n}^{\prime \prime}$ is also presented.

According to Lemmas 4 and 5 in [15] and Theorems 3, 4, and 6 , we can obtain the following theorems.

Theorem 10. Let $A_{n}^{\prime \prime}=\operatorname{SLCirc}\left(\mathfrak{\Omega}_{1}, \ldots, \mathbf{\Omega}_{n}\right)$ be a skew left circulant matrix; then

$$
\begin{array}{r}
\operatorname{det} A_{n}^{\prime \prime}=(-1)^{n(n-1) / 2} \\
\times 2\left[2+t \mathbf{\Omega}_{n}+\sum_{k=1}^{n-2}\left(t \mathbf{\Omega}_{1+i}-\mathfrak{\Omega}_{2+i}\right) x^{n-1-i}\right] \cdot c^{n-2} \\
\quad(n \geq 3),
\end{array}
$$

where $\mathfrak{Q}_{n}$ is the nth sum of Fibonacci and Lucas number.
Theorem 11. Let $A_{n}^{\prime \prime}=\operatorname{SLCirc}\left(\mathfrak{\Omega}_{1}, \ldots, \mathfrak{\Omega}_{n}\right)$ be a skew left circulant matrix for any positive interger $n$; then $A_{n}^{\prime \prime}$ is an invertible matrix.

Theorem 12. Let $A_{n}^{\prime \prime}=\operatorname{SLCirc}\left(\mathfrak{\Omega}_{1}, \ldots, \mathfrak{\Omega}_{n}\right)$ be a skew left circulant matrix; then

$$
\left(A_{n}^{\prime \prime}\right)^{-1}=\frac{1}{l_{n}} \operatorname{SLCirc}\left(y_{1}^{\prime \prime}, y_{2}^{\prime \prime}, \ldots, y_{n}^{\prime \prime}\right) \quad(n \geq 4),
$$

where

$$
\begin{aligned}
& y_{1}^{\prime \prime}=1-\left[(6-4 t) \frac{(-d)^{n-3}}{c^{n-2}}\right. \\
& \left.+\sum_{i=1}^{n-3}\left(\boldsymbol{\Omega}_{n+2-i}-t \mathbf{\Omega}_{n+1-i}\right) \cdot \frac{(-d)^{i-1}}{c^{i}}\right], \\
& y_{k}^{\prime \prime}=-y_{n-k+2}^{\prime} \\
& =\sum_{i=1}^{2}\left(\mathbf{\Omega}_{1+i}-t \mathbf{\Omega}_{i}\right) \cdot \frac{(-d)^{n-k-3+i}}{c^{n-k-2+i}}, \\
& (k=2,3, \ldots, n-2) . \\
& y_{n-1}^{\prime \prime}=-y_{3}^{\prime}=(6-4 t) \cdot \frac{1}{c}, \\
& y_{n}^{\prime \prime}=-y_{2}^{\prime} \\
& =t+\sum_{i=1}^{n-2}\left(\mathbf{\Omega}_{n+1-i}-t \mathbf{\Omega}_{n-i}\right) \cdot \frac{(-d)^{i-1}}{c^{i}} .
\end{aligned}
$$

\section{Norm and Spread of Skew Left Circulant Matrix with the Sum of Fibonacci and Lucas Numbers}

Theorem 13. Let $A_{n}^{\prime \prime}=\operatorname{SLCirc}\left(\mathfrak{\Omega}_{1}, \ldots, \mathfrak{\Omega}_{n}\right)$ be a skew left circulant matrix. Then three kinds norms of $A_{n}^{\prime \prime}$ are given by

$$
\begin{aligned}
& \left\|A_{n}^{\prime \prime}\right\|_{1}=\left\|A_{n}^{\prime \prime}\right\|_{\infty}=\mathfrak{Q}_{n+2}-4, \\
& \left\|A_{n}^{\prime \prime}\right\|_{F}=\sqrt{n\left(\mathfrak{Q}_{n} \mathfrak{Q}_{n+1}-4\right)} .
\end{aligned}
$$

Proof. Using the similar method in Theorem 7, the conclusion is obtained.

Theorem 14. Let $A_{n}^{\prime \prime \prime}=\operatorname{SLCirc}\left(\mathfrak{\Omega}_{1},-\mathfrak{\Omega}_{2}, \ldots,-\mathfrak{\Omega}_{n-1}, \mathfrak{\Omega}_{n}\right)$ be an odd-order alternative skew left circulant matrix; then

$$
\left\|A_{n}^{\prime \prime \prime}\right\|_{2}=\sum_{i=1}^{n} \mathfrak{Q}_{i}=\mathfrak{Q}_{n+2}-4 .
$$

Proof. According to Lemma 2,

$$
\lambda_{j}\left(A_{n}^{\prime \prime \prime}\right)= \pm\left|\sum_{i=1}^{n}(-1)^{i-1} \mathfrak{\Omega}_{i} \omega^{(j-(1 / 2))(k-1)}\right|,
$$


for $j=1,2, \ldots,(n-1) / 2$, and

$$
\lambda_{(n+1) / 2}\left(A_{n}^{\prime \prime \prime}\right)=\sum_{i=1}^{n} \mathfrak{Q}_{i} .
$$

So

$$
\begin{aligned}
\left|\lambda_{j}\left(A_{n}^{\prime \prime \prime}\right)\right| & \leq \sum_{i=1}^{n}\left|(-1)^{i-1} \mathfrak{Q}_{i}(-1)^{i-1}\right| \\
& =\sum_{i=1}^{n} \mathfrak{\Omega}_{i}, \quad\left(j=1,2, \ldots, \frac{n-1}{2}\right) .
\end{aligned}
$$

By (56) and (57), we have

$$
\max _{0 \leq i \leq n+1}\left|\lambda_{i}\left(A_{n}^{\prime \prime \prime}\right)\right|=\sum_{i=1}^{n} \mathfrak{\Omega}_{i} .
$$

Since all skew left circulant matrices are symmetrical, by Lemma 7 in [26], (6), and (58), we obtain

$$
\left\|A_{n}^{\prime \prime \prime}\right\|_{2}=\mathfrak{\Omega}_{n+2}-4 \text {. }
$$

Theorem 15. Let $A_{n}^{\prime \prime}=\operatorname{SLCirc}\left(\mathfrak{\Omega}_{1}, \ldots, \mathfrak{\Omega}_{n}\right)$ be a skew left circulant matrix; then the bounds for the spread of $A_{n}^{\prime \prime}$ are

$$
2 \mathfrak{Q}_{n} \leq s\left(A_{n}^{\prime \prime}\right) \leq\left\{\begin{array}{l}
\sqrt{M-\frac{2}{n} N_{1}^{2}}, \text { if } n \text { is odd, } \\
\sqrt{M-\frac{2}{n} N_{2}^{2}}, \text { if } n \text { is even, }
\end{array}\right.
$$

where $M=2 n\left(\mathfrak{Q}_{n} \mathfrak{Q}_{n+1}-4\right), N_{1}=\mathfrak{\Omega}_{n-1}-\mathfrak{Q}_{-1}, N_{2}=0$.

Proof. Since $A_{n}^{\prime \prime}$ is a symmetric matrix, by Lemma 6 in [26], we get $s\left(A_{n}^{\prime \prime}\right) \geq 2 \max _{i \neq j}\left|a_{i j}\right|=2 \boldsymbol{\Omega}_{n}$.

The trace of $A_{n}^{\prime \prime}$ is, if $n$ is odd, then $\operatorname{tr}\left(A_{n}^{\prime \prime}\right)=\mathfrak{Q}_{1}-\mathfrak{\Omega}_{2}+$ $\mathfrak{Q}_{3}-\cdots+\mathfrak{Q}_{n}=\mathfrak{Q}_{1}+\mathfrak{Q}_{1}+\mathfrak{Q}_{3}+\cdots+\mathfrak{Q}_{n-2}=4+\sum_{i=1}^{n-3} \mathfrak{Q}_{i}$, by (6), we have

$$
\operatorname{tr}\left(A_{n}^{\prime \prime}\right)=\mathfrak{Q}_{n-1}-\mathfrak{Q}_{-1}=N_{1} .
$$

Let $M=2 n\left(\boldsymbol{\Omega}_{n} \mathfrak{Q}_{n+1}-4\right)$; then by Definition 5 in [26], (53), and (61), we obtain

$$
s\left(A_{n}^{\prime \prime}\right) \leqslant \sqrt{M-\frac{2}{n} N_{1}^{2}} .
$$

If $n$ is even, then

$$
\operatorname{tr}\left(A_{n}^{\prime \prime}\right)=N_{2}=\mathfrak{Q}_{1}-\mathfrak{Q}_{1}+\mathfrak{\Omega}_{3}-\mathfrak{Q}_{3}+\cdots-\mathfrak{Q}_{n-1}=0,
$$

hence

$$
\operatorname{tr}\left(A_{n}^{\prime \prime}\right)=N_{2}=0
$$

So the result follows.

\section{Conclusion}

We discuss the invertibility of the skew circulant type matrices with the sum of Fibonacci and Lucas numbers and present the determinant and the inverse matrices by constructing the transformation matrices. The four kinds of norms and bounds for the spread of these matrices are given, respectively.

\section{Conflict of Interests}

The authors declare that there is no conflict of interests regarding the publication of this paper.

\section{Acknowledgments}

The research is supported by the Development Project of Science \& Technology of Shandong Province (Grant no. 2012GGX10115) and the AMEP of Linyi University, China.

\section{References}

[1] D. Bertaccini and M. K. Ng, "Skew circulant preconditioners for systems of LMF-based," in Numerical Analysis and Its Applications, Lecture Notes in Computer Science, pp. 93-101, 2001.

[2] J. R. Claeyssen, M. Davila, and T. Tsukazan, "Factor circulant block matrices and even order undamped matrix differential equations," Matemática Aplicada e Computacional, vol. 3, no. 1, pp. 81-96, 1983.

[3] B. Karasözen and G. Şimşek, "Energy preserving integration of bi-Hamiltonian partial differential equations," Applied and Engineering Mathematics, vol. 26, no. 12, pp. 1125-1133, 2013.

[4] A. Meyer and S. Rjasanow, "An effective direct solution method for certain boundary element equations in 3D," Mathematical Methods in the Applied Sciences, vol. 13, no. 1, pp. 43-53, 1990.

[5] S. J. Guo, Y. M. Chen, and J. H. Wu, "Equivariant normal forms for parameterized delay differential equations with applications to bifurcation theory," Acta Mathematica Sinica, vol. 28, no. 4, pp. 825-856, 2012.

[6] X. Q. Jin, S. L. Lei, and Y. Wei, "Circulant preconditioners for solving singular perturbation delay differential equations," Numerical Linear Algebra with Applications, vol. 12, no. 2-3, pp. 327-336, 2005.

[7] V. C. Liu and P. P. Vaidyanathan, "Circulant and skew-circulant matrices as new normal-form realization of IIR digital filters," IEEE Transactions on Circuits and Systems, vol. 35, no. 6, pp. 625-635, 1988.

[8] M. J. Narasimha, "Linear convolution using skew-cyclic convolutions," IEEE Signal Processing Letters, vol. 14, no. 3, pp. 173-176, 2007.

[9] D. Q. Fu, Z. L. Jiang, Y. F. Cui, and S. T. Jhang, "New fast algorithm for optimal design of block digital filters by skewcyclic convolution," IET Signal Processing, vol. 8, no. 6, pp. 633638, 2014.

[10] H. Karner, J. Schneid, and C. W. Ueberhuber, "Spectral decomposition of real circulant matrices," Linear Algebra and Its Applications, vol. 367, pp. 301-311, 2003.

[11] J. Li, Z. Jiang, N. Shen, and J. Zhou, "On optimal backward perturbation analysis for the linear system with skew circulant 
coefficient matrix," Computational and Mathematical Methods in Medicine, vol. 2013, Article ID 707381, 7 pages, 2013.

[12] P. J. Davis, Circulant Matrices, John Wiley \& Sons, New York, NY, USA, 1979.

[13] Z. L. Jiang and Z. X. Zhou, Circulant Matrices, Chengdu Technology University, Chengdu, China, 1999.

[14] S. Q. Shen, J. M. Cen, and Y. Hao, "On the determinants and inverses of circulant matrices with Fibonacci and Lucas numbers," Applied Mathematics and Computation, vol. 217, no. 23, pp. 9790-9797, 2011.

[15] Y. Gao, Z. L. Jiang, and Y. P. Gong, "On the determinants and inverses of skew circulant and skew left circulant matrices with Fibonacci and Lucas numbers," WSEAS Transactions on Mathematics, vol. 12, no. 4, pp. 472-481, 2013.

[16] Z. L. Jiang, J. J. Yao, and F. L. Lu, "On skew circulant type matrices involving any continuous Fibonacci numbers," Abstract and Applied Analysis, vol. 2014, Article ID 483021, 10 pages, 2014.

[17] X. Y. Jiang and K. Hong, "Exact determinants of some special circulant matrices involving four kinds of famous numbers," Abstract and Applied Analysis, vol. 2014, Article ID 273680, 12 pages, 2014.

[18] Z. Jiang and D. Li, “The invertibility, explicit determinants, and inverses of circulant and left circulant and $g$-circulant matrices involving any continuous Fibonacci and Lucas numbers," Abstract and Applied Analysis, vol. 2014, Article ID 931451, 14 pages, 2014.

[19] S. Solak, "On the norms of circulant matrices with the Fibonacci and Lucas numbers," Applied Mathematics and Computation, vol. 160, no. 1, pp. 125-132, 2005.

[20] A. İpek, "On the spectral norms of circulant matrices with classical Fibonacci and Lucas numbers entries," Applied Mathematics and Computation, vol. 217, no. 12, pp. 6011-6012, 2011.

[21] L. Mirsky, "The spread of a matrix," Mathematika, vol. 3, pp. 127130, 1956.

[22] R. Sharma and R. Kumar, "Remark on upper bounds for the spread of a matrix," Linear Algebra and its Applications, vol. 438, no. 11, pp. 4359-4362, 2013.

[23] J. Wu, P. Zhang, and W. Liao, "Upper bounds for the spread of a matrix," Linear Algebra and Its Applications, vol. 437, no. 11, pp. 2813-2822, 2012.

[24] C. R. Johnson, R. Kumar, and H. Wolkowicz, "Lower bounds for the spread of a matrix," Linear Algebra and its Applications, vol. 71, pp. 161-173, 1985.

[25] Z. Jiang, Y. Gong, and Y. Gao, "Invertibility and explicit inverses of circulant-type matrices with \$k\$-Fibonacci and \$k\$-Lucas numbers," Abstract and Applied Analysis, vol. 2014, Article ID 238953, 9 pages, 2014.

[26] J. Li, Z. L. Jiang, and F. L. Lu, "Determinants, norms, and the spread of circulant matrices with Tribonacci and generalized Lucas numbers," Abstract and Applied Analysis, vol. 2014, Article ID 381829, 9 pages, 2014. 


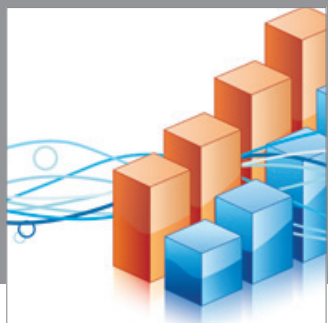

Advances in

Operations Research

mansans

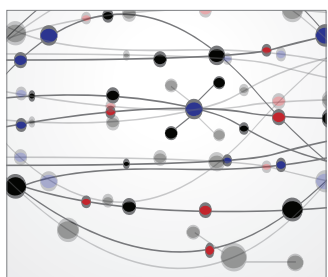

The Scientific World Journal
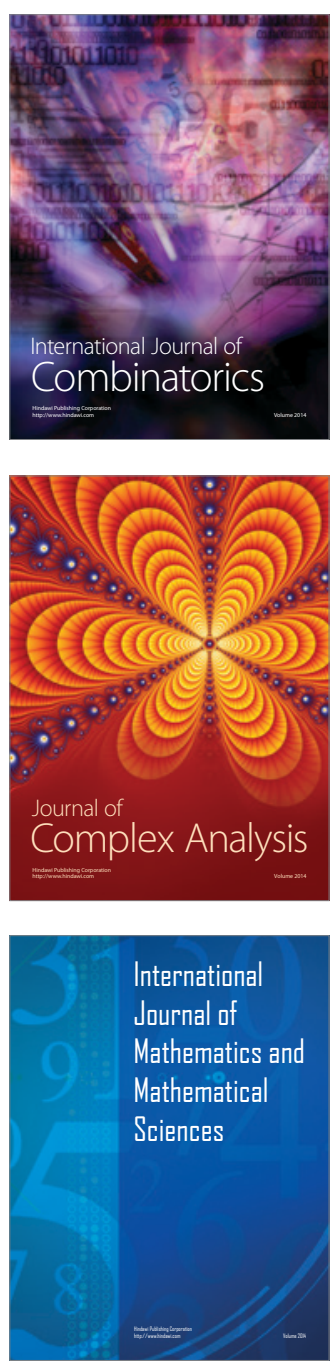
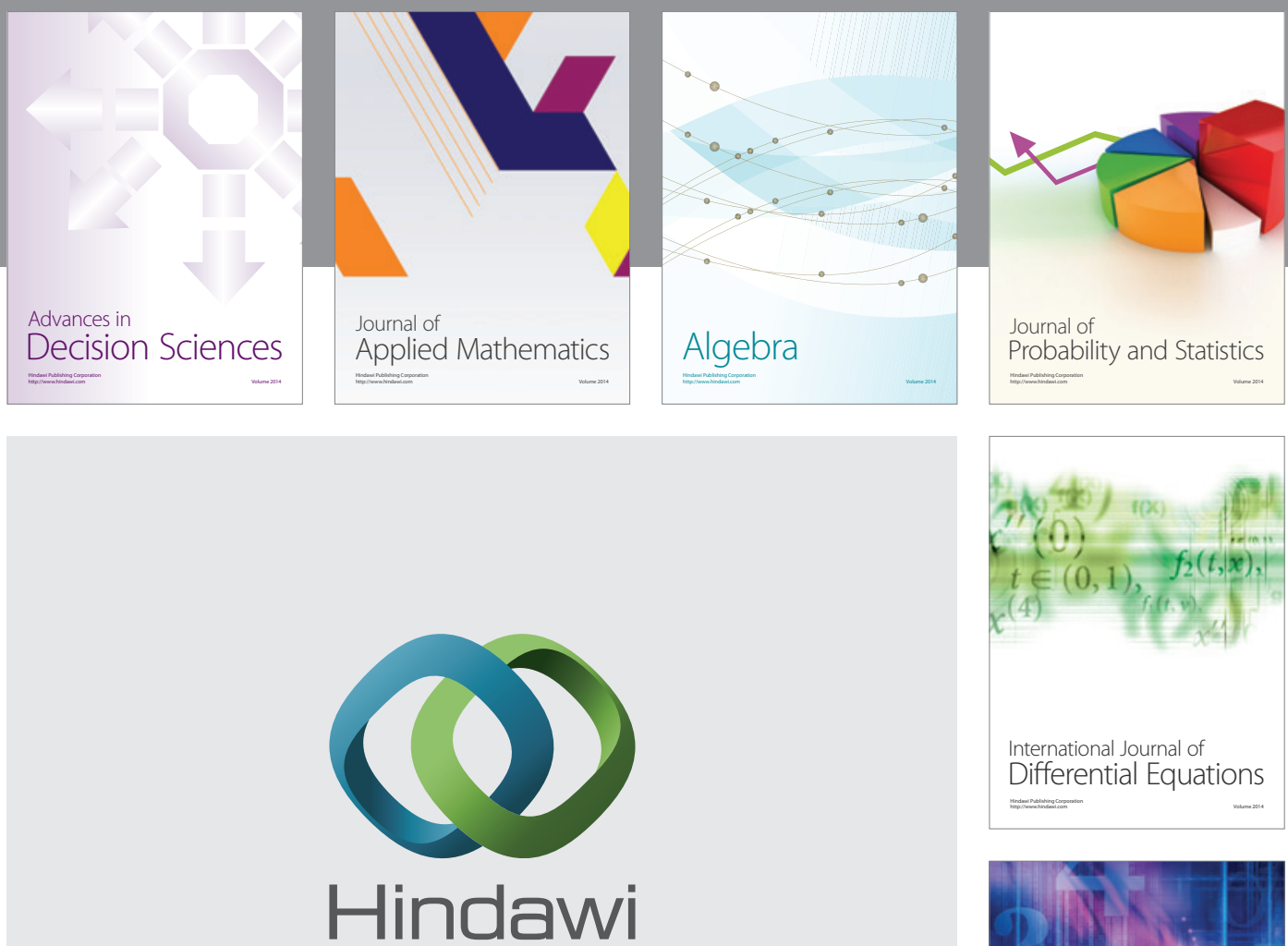

Submit your manuscripts at http://www.hindawi.com
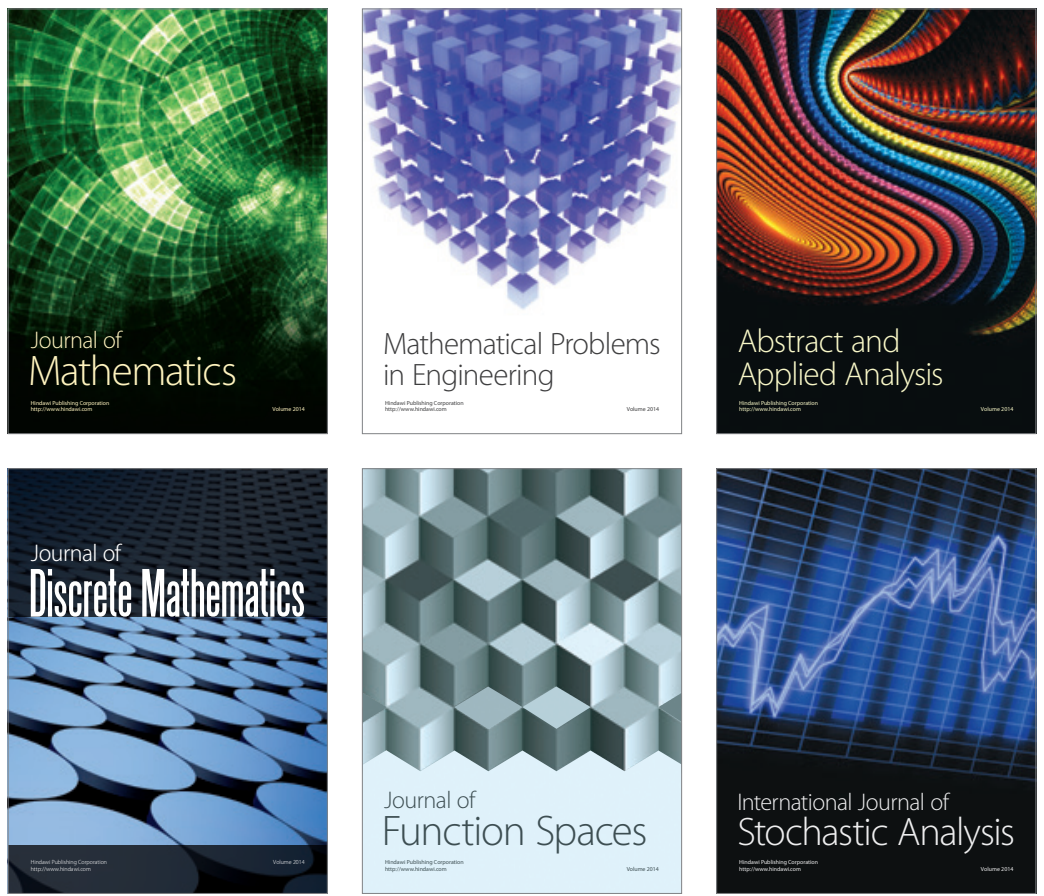

Journal of

Function Spaces

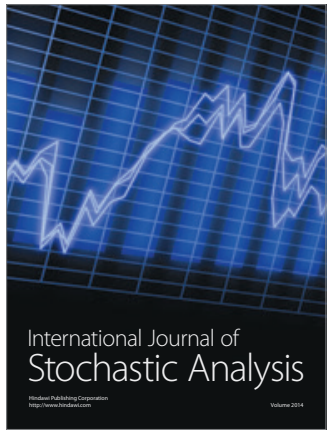

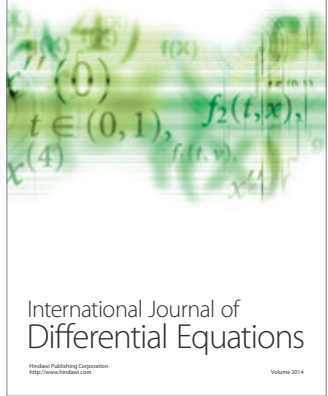
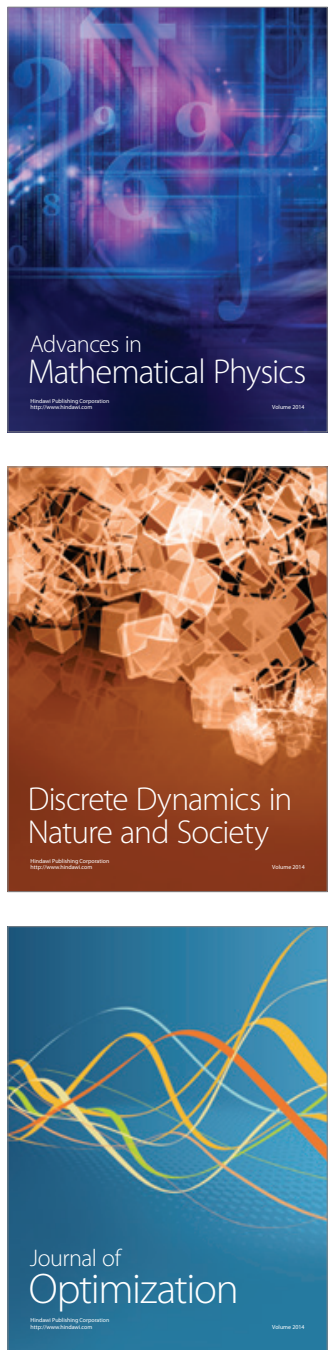